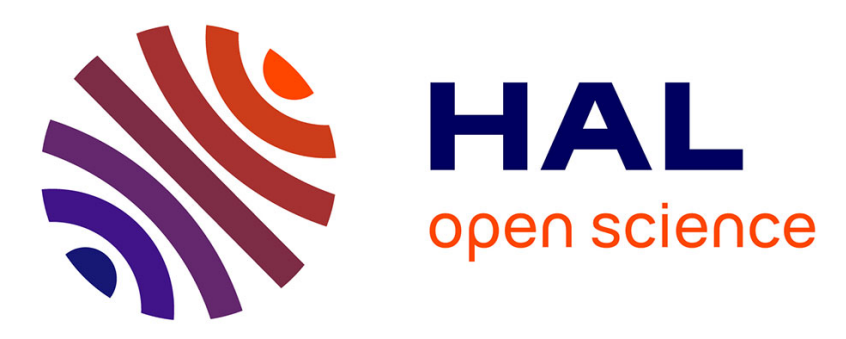

\title{
Noise/Chaos Distinction Applied to the Study of a Fluidized Bed
}

\author{
N. Letaief, C. Rozé, G. Gouesbet
}

\section{To cite this version:}

N. Letaief, C. Rozé, G. Gouesbet. Noise/Chaos Distinction Applied to the Study of a Fluidized Bed. Journal de Physique II, 1995, 5 (12), pp.1883-1899. 10.1051/jp2:1995218 . jpa-00248276

\section{HAL Id: jpa-00248276 https://hal.science/jpa-00248276}

Submitted on 1 Jan 1995

HAL is a multi-disciplinary open access archive for the deposit and dissemination of scientific research documents, whether they are published or not. The documents may come from teaching and research institutions in France or abroad, or from public or private research centers.
L'archive ouverte pluridisciplinaire HAL, est destinée au dépôt et à la diffusion de documents scientifiques de niveau recherche, publiés ou non, émanant des établissements d'enseignement et de recherche français ou étrangers, des laboratoires publics ou privés. 
Classification

Physics Abstracts

$05.45 \mathrm{~b}-47.55 \mathrm{k}$

\title{
Noise/Chaos Distinction Applied to the Study of a Fluidized Bed
}

\author{
N. Letaief, C. Rozé and G. Gouesbet \\ LESP( $\left.{ }^{*}\right)$, INSA de Rouen, BP08, 76131 Mont-Saint-Aignan Cedex, France \\ (Received 4 August 1994, revised 1 February 1995, accepted in final form 18 August 1995)
}

\begin{abstract}
The observation of chaos in fluidization have been claimed by some authors. We analyze the dynamics of a gas-solid fluidized bed and demonstrate that the dynamics is not chaotic but noisy. The random process is studied by using the Hurst analysis in order to look for long-term correlations in the signals. The dynamics may then be modelled by a fractal. Brownian motion process.
\end{abstract}

\section{Introduction}

Though now widely used in industrial processes, fluidized beds are still somewhat not understood from a fundamental point of view. In particular, fundamental approaches are limited by the complexity of the equations involved, and statistical approaches, which are very useful in practical cases, can only give us approximate values. In recent years, many researchers have taken an interest in the chaotic behavior of fluidization. The reason for this interest is probably in part linked to a fundamental property of chaotic systems, i.e. the main features of the underlying dynamics can be retrieved from a single well-chosen time series. Nevertheless, there is no general agreement between experimenters on the subject: Van Den Bleek and Schouten [1] claim that a fluidization process can be characterized by a low-dimensional strange attractor, but Tam and Devine [2] claim exactly the contrary. Such contradictions may be due to the fact that phenomena grouped under the same vocable "fluidization" can actually take quite different forms (slugging or bubbling, etc...), and, therefore, the presence or absence of chaos may sensitively depend on the actual experiments under study.

But, on the other hand, as emphasized by Provenzale et al. [3], it is now recognized by many experimenters that one method is not sufficient to establish evidence for chaos, and that the simultaneous use of several methods can be required to avoid incorrect conclusions [4]. For instance, as far as the evaluation of correlation dimension is concerned, Rapp et al. [5] showed that filtered noise can seemingly behave as low-dimensional attractors and the same phenomena have been observed by us with an autoregressive random process. Therefore, invoking a measured small correlation dimension to conclude about the existence of low-dimensional chaos may be misleading. With other methods, such as the computations of the Lyapunov

(*) URA CNRS 230-CORLA 


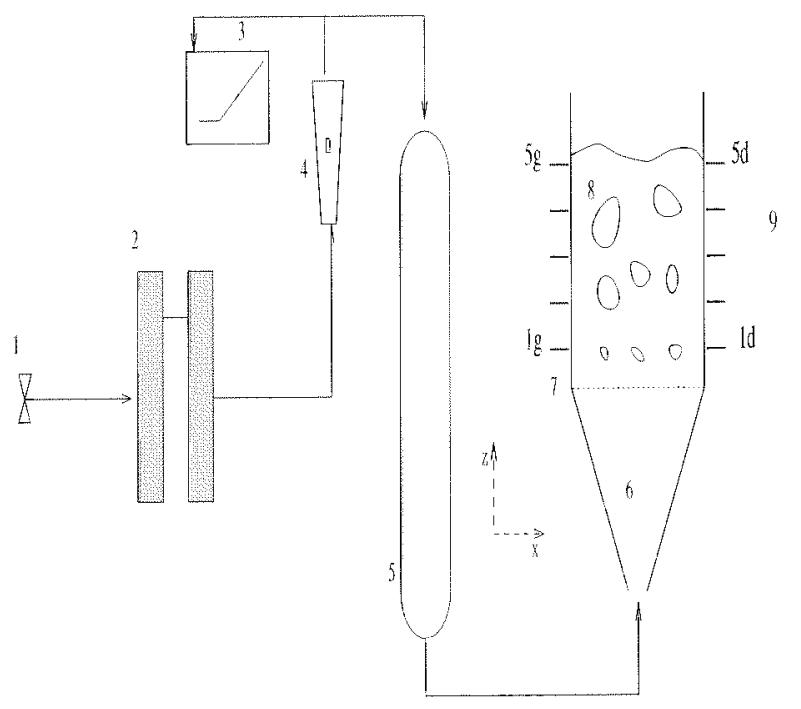

Fig. 1. Experimental set-up. (1) air supply, (2) sub-micronic filters, (3) rotameter, (1) mercury, (5) vacuum chamber, (6) dessication, (7) distributor, (8) fluidization, (9) pressure taps.

exponents (or even of the largest Lyapunov exponent), experimental conditions can prevent the algorithms working, and do not give any interpretable results in many real cases (for which noise is important). Thus, the correct attitude when dealing with problematic data is to examine experimental results by several means, using a battery of tests, before establishing a diagnosis of the chaotic or random nature of the data. Obviously, this heuristic attitude is very different from that in the case of well-known chaotic data.

Our aim, in this paper, is to determine the nature of signals recorded from a fluidized bed, and, in particular, to establish whether our data result from a chaotic process or not. We therefore use several methods of investigation, and among them, the method of surrogate data, discussed by Theiler [6] in 1992. This method allows systematic comparisons between noise and chaos. Using this method as well as other more classical methods, we will conclude that the data from our fluidized bed are not the results of a low-dimensional chaotic system.

The paper is organized as follows. In Section 2, the experimental set-up and the basic features of measured signals are presented. Section 3 is devoted to the description of noise/chaos distinction methods, and to their application to some well-known benchmark examples. Section 4 is devoted to the applications of the methods of distinction between noise and chaos to our particular case. Section 5 describes the Hurst analysis, which is a method of characterization of the long-term behavior of signals, and its application to the experimental data. Section 6 is a conclusion.

\section{Description of the Experiments}

2.1. Expertmental Set-up. - The set up is represented in Figure 1. The fluidized bed is two-dimensional: its inner dimensions are $300 \times 500 \times 10 \mathrm{~mm}^{3}$. The walls are in plexiglas, to allow visual observation. The fluidization gas employed is air, filtered and dried beforehand in a dessication column. The parameter is the flow rate of air divided by the distributor area, also called superficial velocity. The distributor is a porous medium made out of bronze, thus 
ensuring a regular distribution of the air flow. The static depth of the particle bed is about $240 \mathrm{~mm}$. The fluidized particles are thin glass balls, their diameter being between 100 and $200 \mathrm{~m}$. We thus have a deep bed (by opposition to shallow beds), belonging to the group $\mathrm{B}$ of Geldart's classification.

Two specific values of the superficial velocity are important here: the minimum fluidization velocity $\left(v_{\mathrm{mf}}\right)$, which corresponds to the state where the drag forces balance the weight of the particles, and the minimum bubbling velocity $\left(v_{\mathrm{mb}}\right)$, corresponding to the appearance of the first bubbles. For the group of beds here considered, $v_{\mathrm{mf}}$ is nearly equal to $v_{\mathrm{mb}}(\approx 0.05 \mathrm{~m} / \mathrm{s})$, i.e. we cannot in practice distinguish between the incipient fluidization and the subsequent onset of bubbling instability which appears for an extra very small increase of the control parameter. This means that one cannot expect to explicitly observe a road towards chaos. On the contrary a fully developed chaos (or random process) is reached almost immediately.

After their apparition, bubbles grow by coalescence as they rise, this coalescence process having been previously extensively investigated [8].

2.2. Meastred Quantitues. - In a first step, bubbling being identified as a fundamental process in the bed, we measured a quantity directly related to this process. Then, we recorded the appearance and evolution of bubbles at a given point of the bed referenced by its coordinates $(x, z)$ (Fig. 2). A laser beam source and a photodetector are placed in front of each other. When bubbles pass in front of the detector, the laser beam attenuation is small, and the corresponding photodetector signal is high. Otherwise, it is practically equal to zero, leading to an essentially binary signal, as shown in the figure (after use of a threshold level). From such a signal, we may determine the transit time $t_{\mathrm{p}}$ of bubbles and the delay time $t_{\mathrm{c}}$ between bubbles. The whole procedure has been validated by filming the bed, simultaneonsly recording the photodetector signal, and comparing the characteristic times $t_{\mathrm{p}}$ and $t_{\mathrm{c}}$, measured from both the signal and the film. Here it will suffice to say that the analysis of time series built from successive $t_{\mathrm{p}}$ 's or successive $t_{\mathrm{c}}$ 's leads to the unambiguous conclusion that the dynamics is random without any indication of any deterministic components [9].

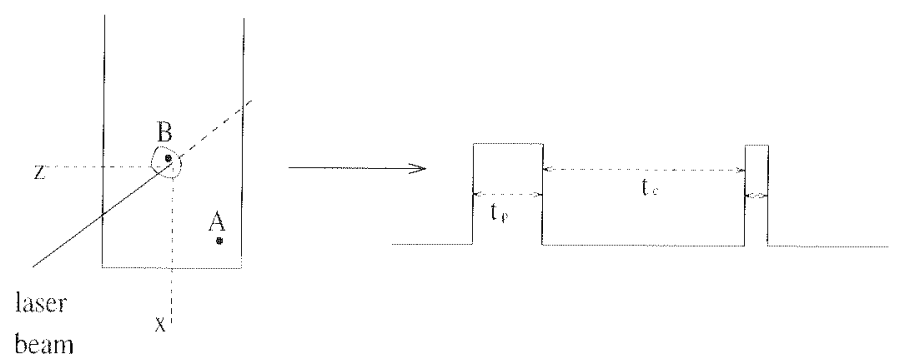

fluidized bed

signal in the time domain

Fig. 2. - Emergence and vanishing time recording of bubbles.

This conclusion should however be softened by considering the following items :

i) Measurements based on bubble characterizations are essentially local and, by introducing some kind of spatial averaging, we may wonder whether a global deterministic component could not be detected. 
ii) Analyzed time series are discrete and thresholding may have introduced spurious effects and spoilt some relevant information.

Therefore, it has been decided to measure quantities being both global (i.e producing some spatial average) and time continuous, namely pressures. Taps have then been inserted laterally on each side along the fluidized bed (Fig. 1), so that either the pressure at a given altitude in the set-up, or the difference of pressure between two given points could be measured. Pressure measurements are carried out by using a membrane pressure sensor, with a precision of $0.2 \%$ and a time response of $10 \mathrm{~ms}$. Signals are sampled and recorded by using a Sun 4 SPARC workstation. We observed that there was no significant frequency above $50 \mathrm{~Hz}$ in the pressure dynamics. Therefore, as indicated by Shannon's theorem, sampling frequency should be greater than or equal to $100 \mathrm{~Hz}$. A sampling rate equal to $200 \mathrm{~Hz}$ has then be chosen, producing smooth curves, without any apparent noise at the visual level.

2.3. ExPERImental Results. - Fan et al. [10] have experimentally demonstrated that the pressure fluctuations at a given altitude were due essentially to bubbles crossing at this altitude. the contribution from distant bubbles being less important than that from neighbouring ones. We can thus estimate that the recorded signals correspond to global measurements of the phenomenon, and is representative of the experimental setmup, with a spatial averaging on an horizontal section.

Typical examples of recordings are shown in Figure 3, for two superficial velocities: the smallest one is just a little larger than the experimental minimum of fluidization velocity, and the other corresponds to more than twice that velocity. The only observation possible is the irregularity of the signal, whatever the superficial velocity. The Fourier transforms of the time series are broad-band (Fig. 4), and they are typical of fluidization [11-14]. They also present strong similarities with the spectra obtained for a bubble column [15] (where the particles are replaced by a liquid medium).

One can define a characteristic frequency by averaging all the frequencies corresponding to amplitudes greater than $80 \%$ of the total integrated amplitude of the spectrum. This characteristic frequency increases with the gas velocity. This is related to the fact that although

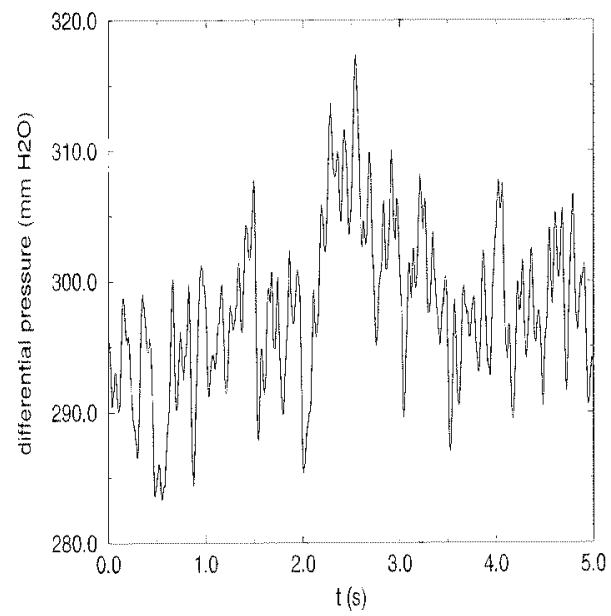

a)

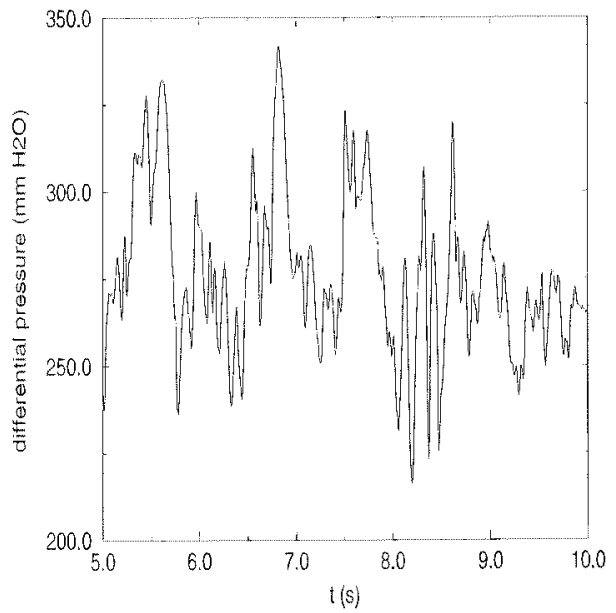

b)

Fig. 3. Left-hand differential pressure recordings for two superficial velocities. 


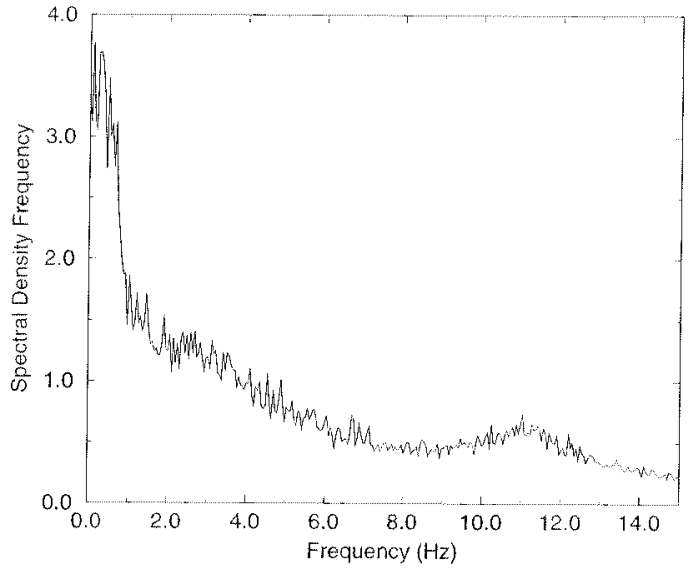

a)

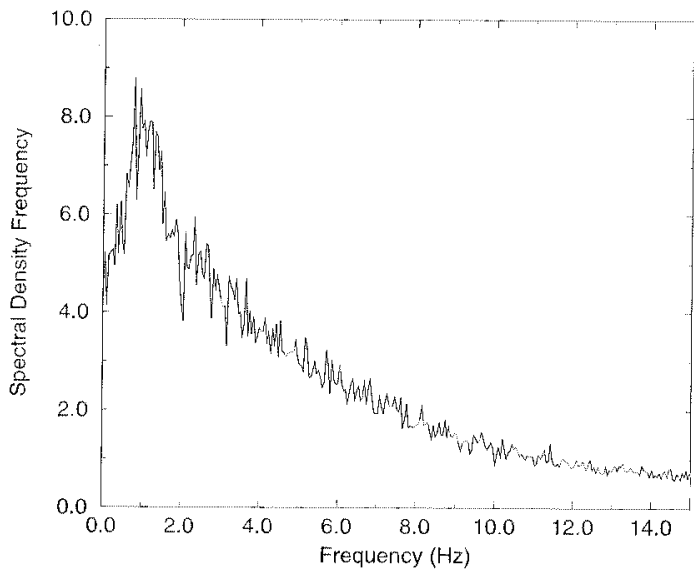

b)

Fig. 4. - Left-hand differential pressure for two superficial velocities: Fourier's spectra.

bubbles diameters increase with gas velocity, their velocity also increase, eventually leading to higher frequencies.

From a time series $\left(x_{i}\right)$, we reconstructed a trajectory $\left(X_{i}\right)$ using the method of delays, with a lag time $p$, in a reconstructed phase space of dimension $n$. Presented results correspond to a sampling time of $5 \times 10^{-3} \mathrm{~s}$, to be compared with the inverse of the characteristic frequency which is about $5 \mathrm{~s}$. For the dimension calculations later presented, the sampling time has been varied, i.e. increased up to $2 \times 10^{-2} \mathrm{~s}$ without modifying the results. Conversely, a sampling time of $5 \times 10^{-3} \mathrm{~s}$ may be too short to analyze forecasting errors (see beginning of Sect. 4 ).

In a three-dimensional phase space, the reconstructed trajectory (Fig. 5) is nearly twodimensional. The two-dimensional character of the object is actually not apparent in the figure, but becomes evidenced if it is rotated on a screen using a graphical AVS system. We did not detect any periodic orbits nor other regular structures like recurrences, i.e. the trajectory seems essentially desorganized.

Also, in agreement with the two-dimensional character of the object exhibited in Figure 5 , we evaluated correlation dimensions using the Grassberger-Procaccia algorithm (see [16] and many references in [17]), which we found to be equal to about 2 . This fact is however not sufficient to conclude to the presence of a low-dimensional attractor. In particular, we reproduced similar results by using the Grassberger-Procaccia algorithm on a time series generated by autoregressive noise. Therefore, a stochastic process analyzed by evaluating correlation dimensions may lead to the spurious identification of deterministic chaos. Such a conclusion has previously been also pointed out by Rapp et al. [5] by analyzing time series generated by a filtered noise. Due to such a fact, it is likely that some claims about the presence of chaos in experimental signals, including fluidization, could be incorrect. In some cases, a most reliable conclusion must therefore be obtained by using noise/chaos distinction techniques exposed in the next section.

\section{Noise/Chaos Distinction Methods}

Two techniques are discussed in this section. The first one, relying on the discussion of forecasting errors, has been developed by Farmer and Sidorowich [18] and was used by Sugihara 


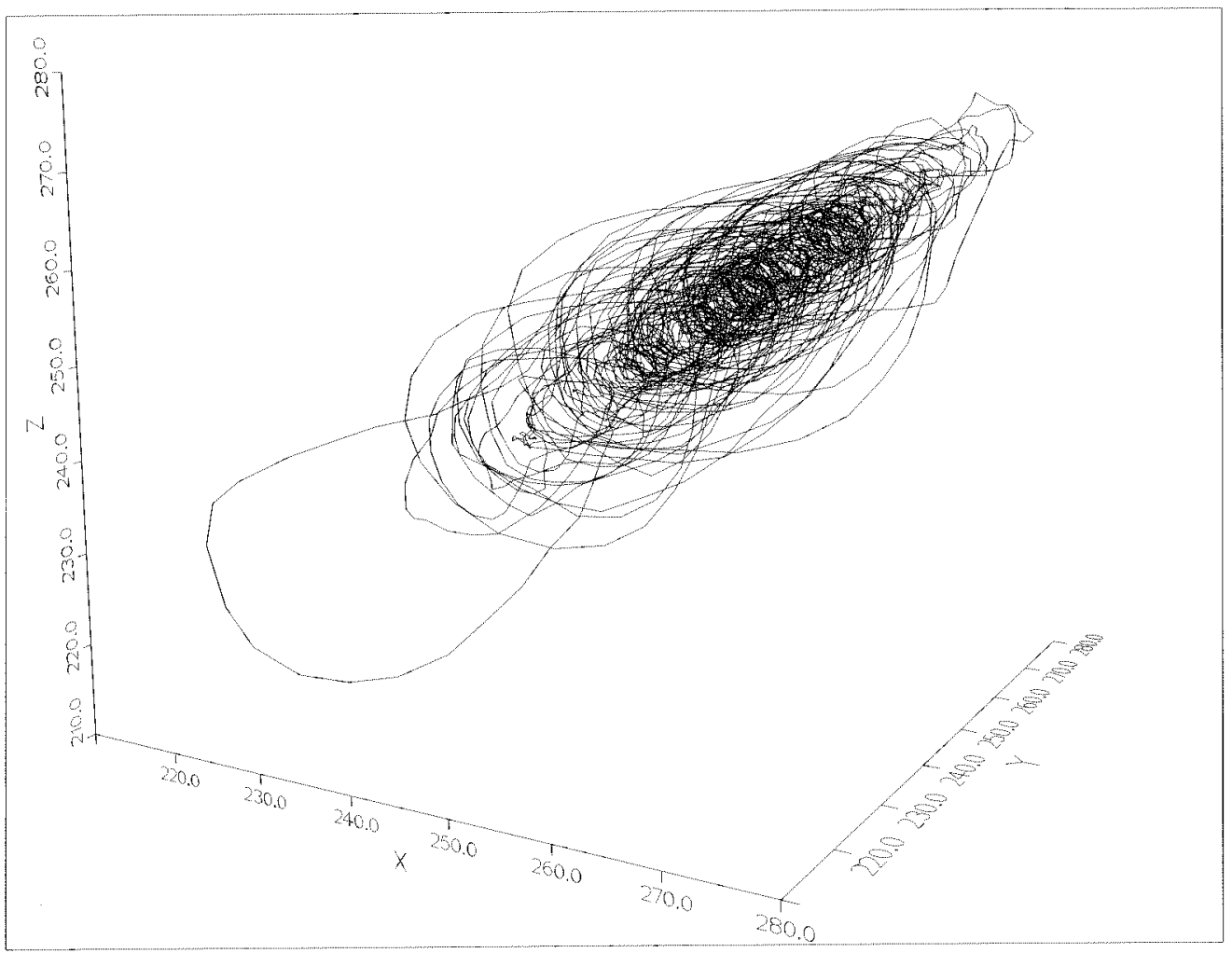

Fig. 5. - 3D trajectory reconstructed by the delay method, $p=3, u=0.09 \mathrm{~m} / \mathrm{s}$, for a measurement of absolute pressure at point 2 , at the right-hand side of the bed.

and May [19] to analyse biological time series, and also by Wales [20] to extract the largest Lyapunov exponent from a time series. The second one, the surrogate data method, was exposed by Theiler et al. [6].

Both methods only require the knowledge of a scalar time series and are therefore well adapted to the analysis of experimental signals when only one variable is recorded versus time.

3.1. Forecasting Errors. - Given a time series $\left(x_{i}, i=1, \ldots n\right)$, the $n_{1}$ first points of the series are used to predict $n_{2}$ other points. The prediction principle is as follows.

A trajectory is constructed by the method of delays (parameters $n, p$ ). Given a reference point, $X_{t}$, we estimate a value $\hat{x}_{t+T}$ of the first coordinate $x_{t+T}$ of the point $X_{t+T}$ at time $t+T$, in which $T$ is the time evolution given as multiples of $\delta t$. Values of $T$ are small when compared to characteristic times of the dynamics. This is achieved by selecting the $m$ closest neighbours $X_{1}, \ldots, X_{m}$ of $X_{t}$, and by searching for the linear map $L$ which transforms $X_{i}$ to $x_{i+T}$. for $i=1, \ldots, m$. The coefficients of the map are found by linear regression (we used a singular value decomposition technique $[27, \mathrm{pp} .5263]$ ), and $\hat{x}_{t+T}$ is then taken as the image of $X_{i}$ under $L$.

The forecasting error $E(T)$ is afterwards given by the square root of the averaged sum of the $n_{2}$ values of $\left|\hat{x}_{t+T},-x_{t+T}\right|^{2}$, divided by the standard deviation $\sigma_{x}$ of the $n_{2}$ points $x_{j+T}$, 
so that the forecasting error magnitude does not depend on the signal's scale:

$$
E(T)=\frac{\sqrt{\left.\left(<\left(\hat{x}_{t+T}-x_{t+T}\right)^{2}\right\rangle\right)}}{\sqrt{\left.<(x-<x>)^{2}\right\rangle}}
$$

where the brackets denotes the average on the $n_{2}$ forecasted points.

The behaviour of the forecasting error gives us an indication on the nature of the time series studied: a forecasting error which increases exponentially with the forecasting time is a signature of deterministic chaos, whereas a forecasting error which oscillates around a constant is almost certainly the indication that the time series is uncorrelated noise.

Though interesting and easy to implement, this method has severe limitations, because it does not allow any distinction between a stochastic process having strong correlations, also called colored noise, and deterministic chaos. However, by using a very sophisticated prediction technique, let us mention that it is possible [21] to discriminate between colored noise (powerlaw behaviour), and chaos (exponential behaviour).

3.2. The Method of Surrogate Data. - In this method, the studied time series is compared with $N$ surrogate time series, which are stochastic time series constructed to possess some basic features (to be defined) shared with the original one. The principle of the test is that if the studied series and the surrogate ones compare favorably in a sense to be defined later, then it is reasonable to affirm that the studied time series is random. It can then be viewed as a realization of the same random process that had generated the surrogate data. Therefore, if we compute a number representative of each time series (the original and each surrogate ones), the number computed from the original time series must be a realization of the random process generating the numbers computed from the surrogate series.

In practice, the number computed for each time series is called the discriminating statistic of the test. It is noted $Q_{\mathrm{D}}$ for the original time series, while $Q_{\bar{H}}$ will denote the average of the $N$ numbers calculated from the $N$ surrogate ones, with $\sigma_{H}$ the standard deviation. The difference between the studied time series and the surrogate ones is then quantified by a critical ratio, which is given by:

$$
\mathrm{RC}=\frac{\left|Q_{\mathrm{D}}-Q_{H}\right|}{\sigma_{H}}
$$

A very large critical ratio expresses a wide difference between the discriminating statistics of the original time series, and those of the surrogate ones, i.e. a poor comparison between them. In this case, when $Q$ is the forecasting error, we reject the hypothesis that the data are stochastic, and decide that they are chaotic. On the contrary, for small values of the critical ratio, the null hypothesis denoted $H_{0}$ has to be accepted, i.e. the original data are stochastic. From the statistical test theory, the threshold value of the critical ratio is taken equal to 2. The null hypothesis is then accepted for critical ratios smaller than 2 and otherwise rejected. With the chosen threshold value, the probability of being wrong, i.e. rejecting $H_{0}$ (data are rejected chaotic) whereas $H_{0}$ is true (data are actually chaotic), is equal to $5 \%$.

The first step of the method is then to construct $N$ random data sets which closely mimic the tested set. There we used a method suggested by Theiler et al. [22], taking for the surrogate data time series having the same Fourier spectrum than the original set, with phases shuffled in the Fourier transform. The resemblance is ensured by the fact that the original and surrogate time series possess the same Fourier spectrum, and thus the same autocorrelation function. but the randomization of the phases of the Fourier transform implies the stochasticity of the surrogate data. We fixed the number of surrogate data sets at $M=40$, ensuring a good enough statistical precision. 
The discriminating statistic of the test might be any number representative of the time series. Nevertheless, the use of numbers representing a signature of the non-linearity of the data is obviously highly recommended. Our choice has been the forecasting error which has the advantage of giving a noise/choas distinction test by itself (previous subsection) and which is furthermore not computer time-consuming. Other choice could have been a correlation dimension or a Lyapunov exponent but the significance of these quantities for the surrogate random signals would be doubtful.

Examples of results are now given for some benchmark numerically generated time series i) from Lorentz and Rössler chaotic attractors for the deterministic chaotic case, and ii) from autoregressive processes for the random case. In Figure 6 devoted to the Lorentz system, the forecasting error increases far more slowly for the tested series than for the surrogate series, and consequently the critical ratio is high, equal to or greater than 15 , much larger than the threshold value of 2 . Therefore, the null hypothesis $H_{0}$ is rejected and the processed data are chaotic, as expected.

Conversely, Figure 7 displays the case of a 5-degree autoregressive noise. Then the forecasting errors are close for both the original and the surrogate data. The critical ratio is smaller or not significantly bigger than 2 , and we may conclude that the original series is generated by a. random process.

3.3. Influence of PARAmeters. - The number of surrogate sets $M$ has been fixed equal to 40 . This choice is a compromise between statistical accuracy requirement and CPU timeconsumption requirements. Nevertheless, since we use the forecasting error method to compute the discriminating statistic, the influence of its parameters has to be investigated. We found that when $n_{1}$ and $n_{2}$ were sufficiently large to ensure statistical precision, a change in the $n_{2}$ value has little influence on the result. But $n_{1}$ must be large enough to be representative of the attractor, and its value thus depends on the sampling rate. For very regular structures, a small number of pseudo-periods is sufficient to ensure realistic precision. We increased the number of points when working on experimental attractors, because of the increasing complexity of the latter.

We will not extensively discuss the role of the parameters $n$ and $p$ : it is essentially the same as for the correlation dimension computation, and is thus discussed by several authors [23 26]. As a brief summary, let us recall that the embedding dimension must be large enough to unfold the attractor in the embedding space (Takens criterion). However, the precision of the computation is affected when the embedding dimension is too large, i.e. we have to search for an optimal $n$. Also, the lag time $p$ must be chosen large enough to avoid the trajectory collapsing on the diagonal, but not too large, otherwise decorrelation of the trajectory vector components will occur. The optimal parameters were found through a trial and error process.

Furthermore, there must be enough neighbours $m$ to warrant the accuracy of the computation of the forecasting error. Because of the regression technique used (singular value decomposition), $m$ must be greater than the embedding dimension $n$. On the other hand, we do not possess any theory allowing us to determine an optimal value for $m$. It can just be said that the greater the number of neighbours, the greater the accuracy of forecasting, but that taking too many neighbours will lead us to consider as neighbours points of the trajectory which are in reality quite far from the reference point and will spoil the computation of the forecasting error, i.e here again we have to search for an optimal value. This effect can be seen in Figure 8 where we have indicated the forecasting error, for the Lorentz system, for different values of $m$, the other parameters being kept constant. An optimum (i.e. a minimum) of the forecasting error is obtained for $m=50$. Nevertheless, though the value of $m$ influences the forecasting error, the repercussion of this influence on the result of the test is negligible. In 

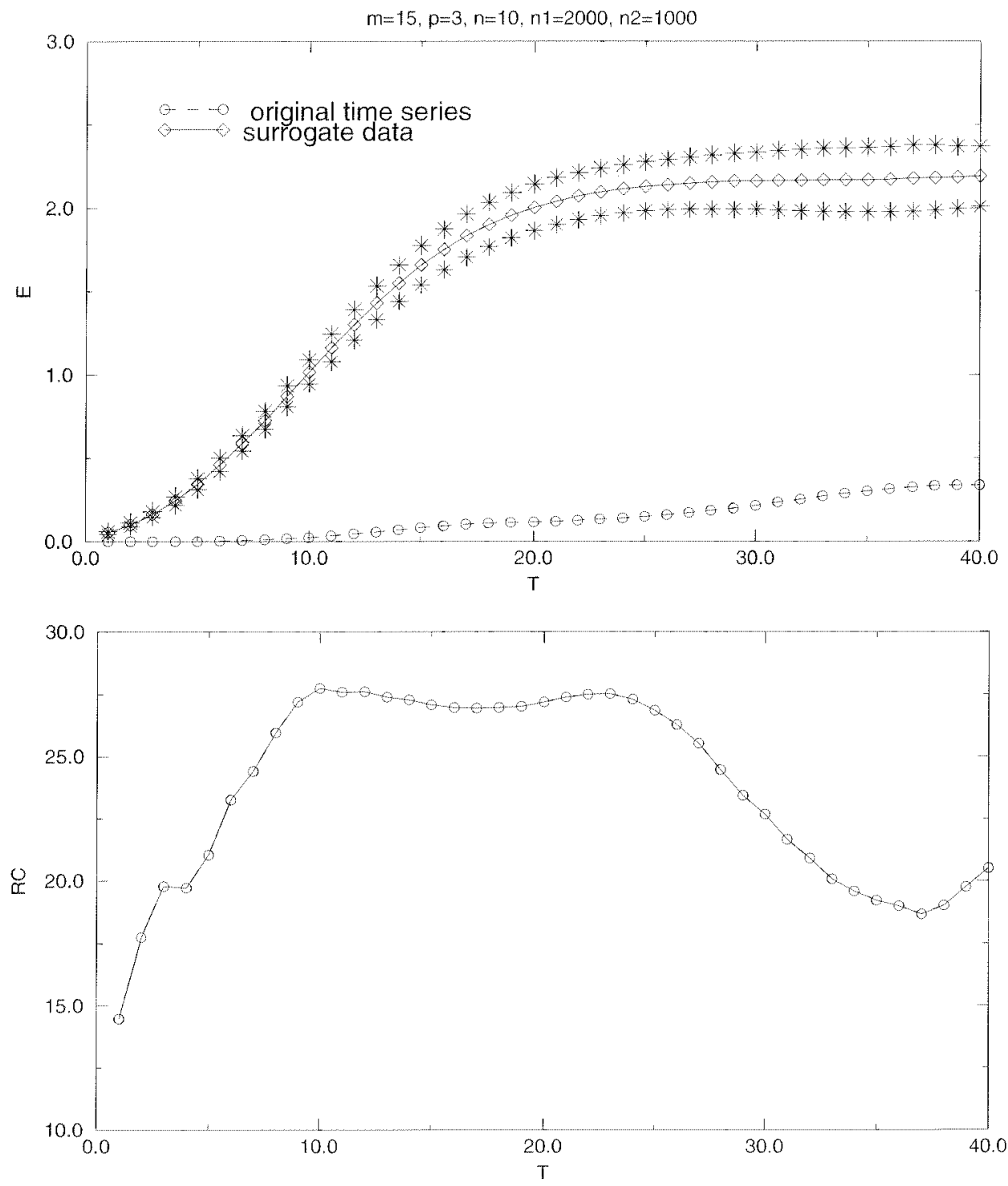

Fig. 6. Forecasting error, and critical ratio, for the Lorentz process, errors bars are represented by the symbol $(\star)$. $T$ is the time evolution given as multiples of $\delta t$.

fact, the effects are quite similar on the forecasting errors of both the studied signal and of the surrogate data, so that the comparison is not significantly modified by the parameter $m$. For instance, we have tested several values of $m$ for the Lorentz process. Whatever $m$, the null hypothesis $H_{0}$ is rejected, i.e. the Lorentz process was found to be chaotic. Conversely, the same test applied to an autoregressive noise always led us to the acceptance of the null hypothesis $H_{0}$, regardless of the value of $m$, i.e. the process was found to be random.

We also investigated the effect of noise in the case of the Lorentz system, by modifying time series with additive noises of various amplitudes $(2,5$, and $50 \%$ of the amplitude of the 

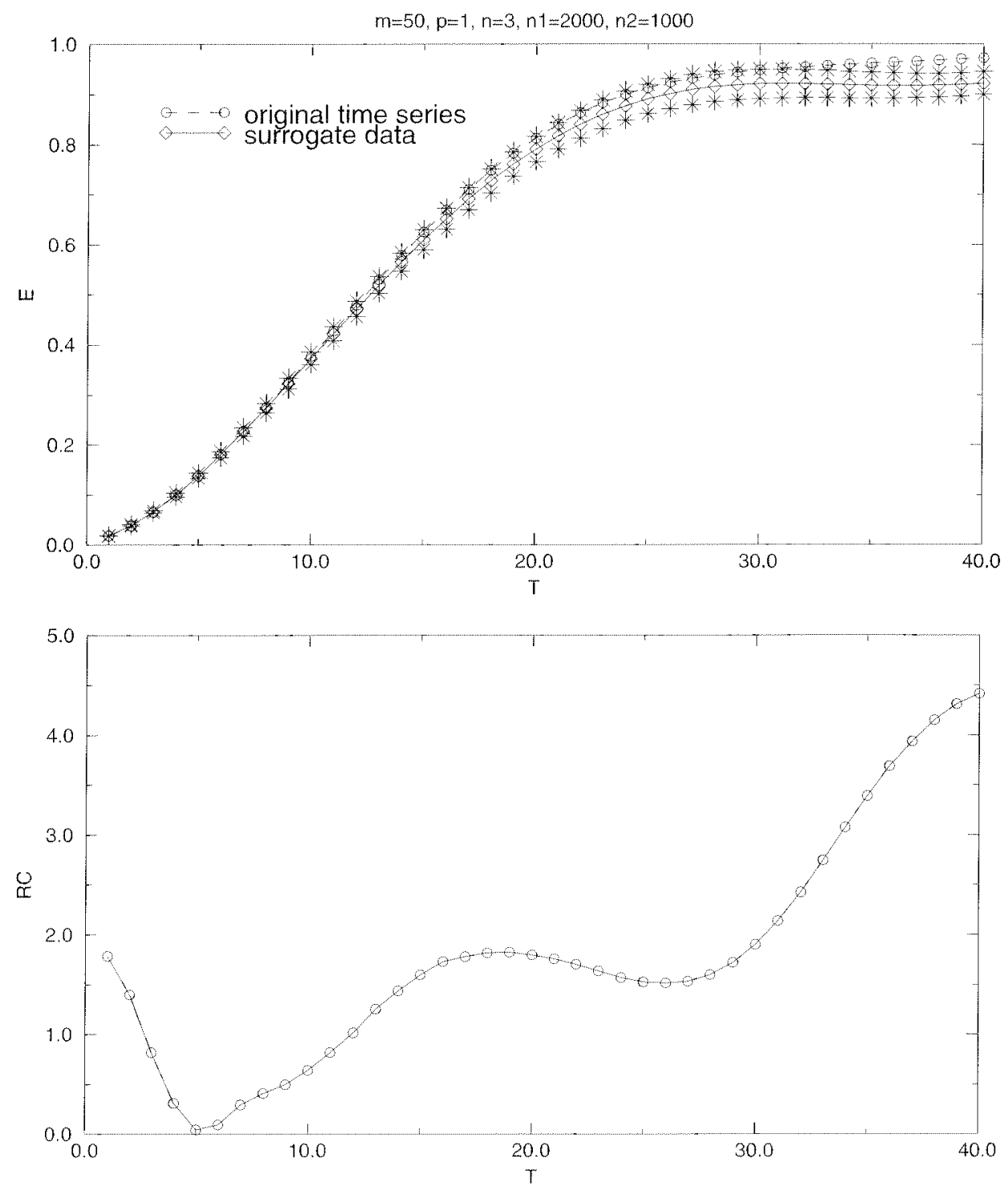

Fig. 7. Forecasting error, and critical ratio, for a 5-degree antoregressive noise.

process). We fonnd that the surrogate data method did not work in the third case, but was still valid for the first two cases, which correspond to many experimental situations. Thus, the method of surrogate data can be considered as reliable and robust with respect to noise and changes in the parameters values.

\section{Application to the Experimental Series}

A typical example of the results is displayed in Figure 9. The difference of forecasting error between experimental and surrogate data is small, leading us to the conclusion that the data is 


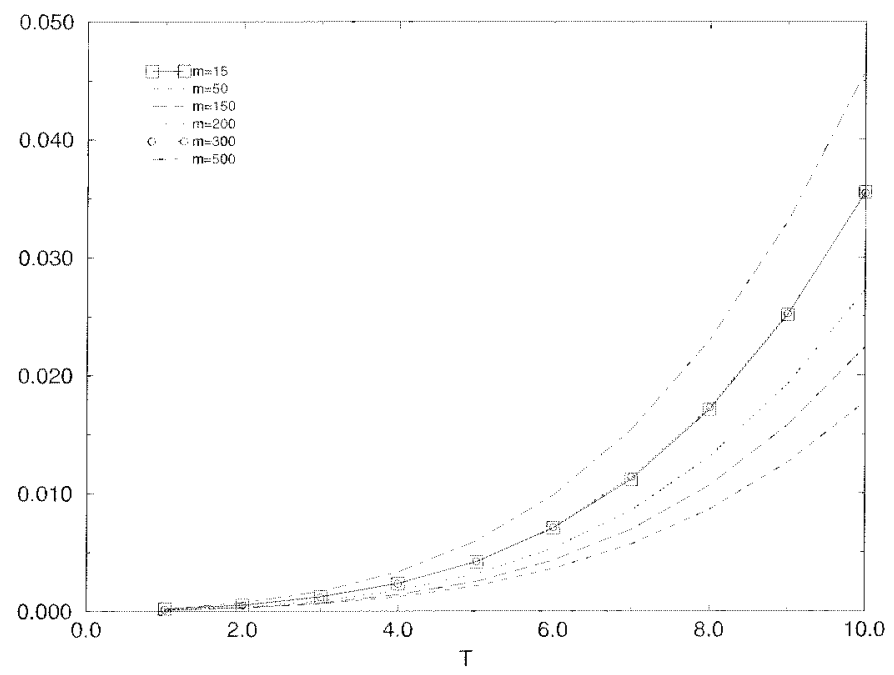

Fig. 8. - Forecasting error computed for the Lorentz process, for different numbers of neighbours $m$. $n=10 ; p=3 ; n_{1}=2000 ; n_{2}=500$.
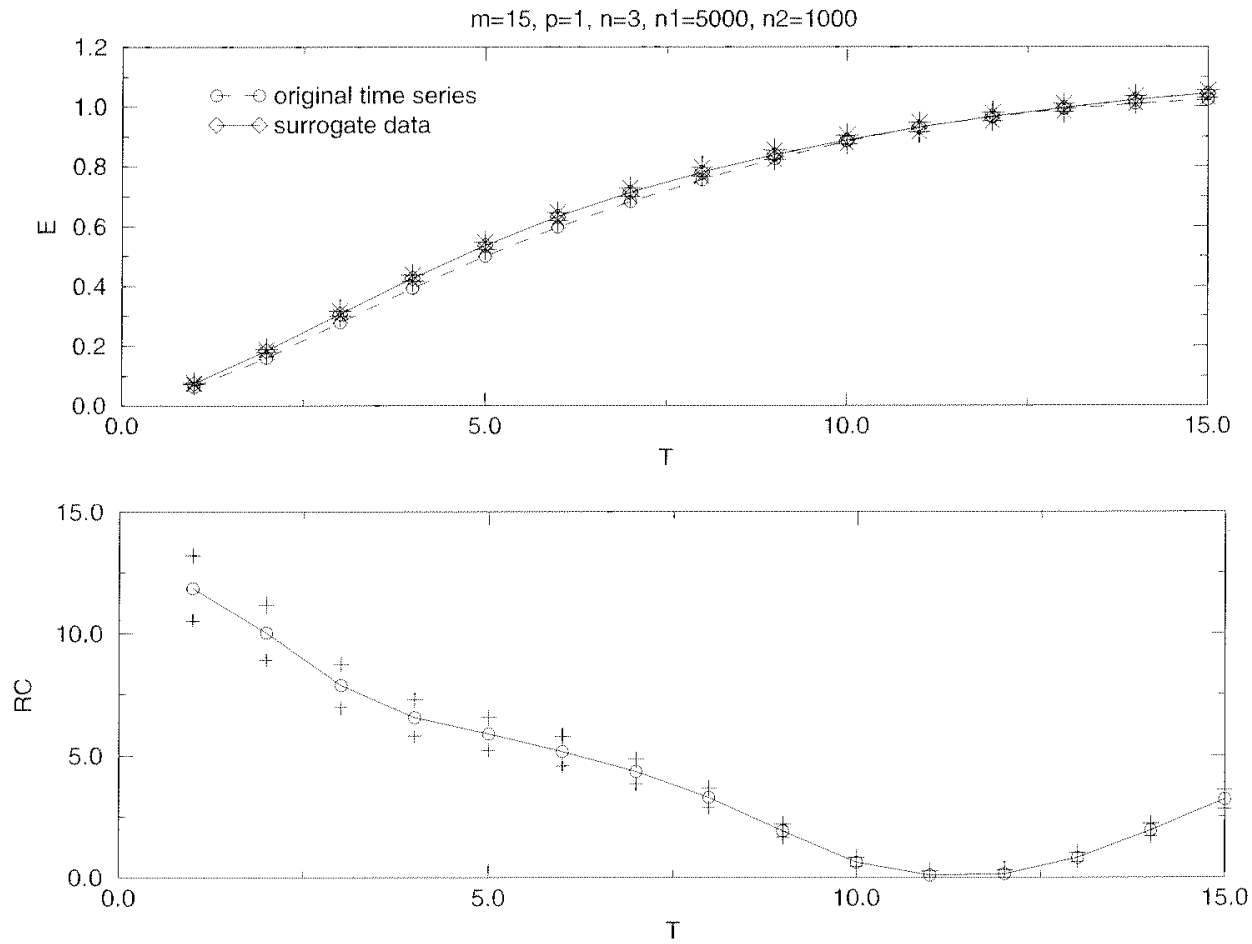

Fig. 9. - Forecasting error, and critical ratio, for a recording of absolute pressure. on the left, at the tap number two, with a superficial velocity $u=0.12 \mathrm{~m} / \mathrm{s}$. 

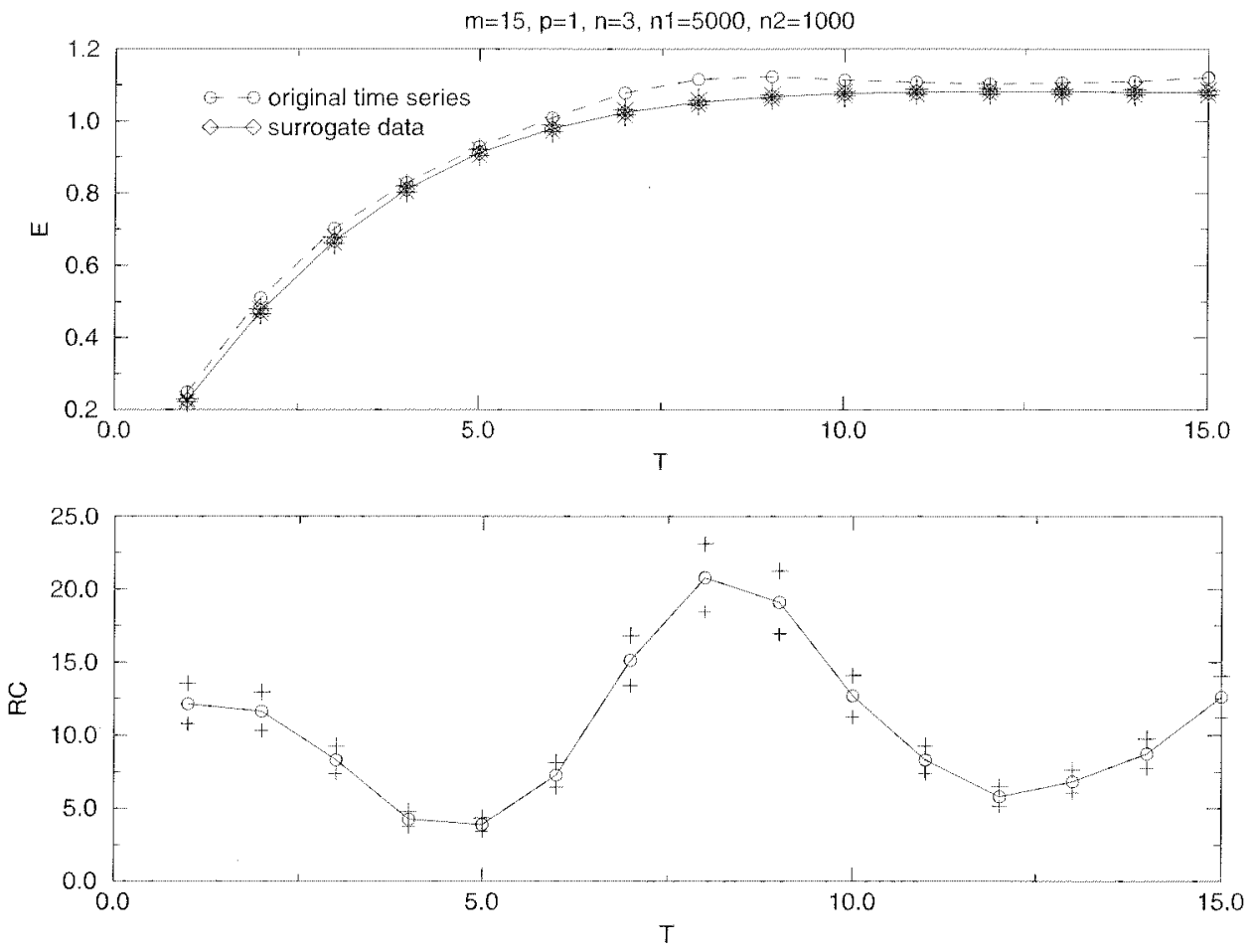

Fig. 10. Forecasting error, and critical ratio, for the same recording, sampled at every second point.

not produced by a low-dimensional chaotic system. But, on the other hand, the critical ratio corresponding to this case is large, due to a very small dispersion around the average for the surrogate data error (denominator in relation (2)). Strictly speaking, we ought to reject the null hypothesis and claim for chaos, but the conflict between both conclusions must lead us to refine our analysis.

Let us remove every second point from the initial time series. In this way, the sampling rate is now divided by two. We apply the method of surrogate data on this new time series, without modifying any parameter. Then, the whole procedure is repeated, so, at the next step, the time series is numerically sampled at a rate which is four times smaller than the initial one

The results we obtained for the time series sampled at every second point show a forecasting error for the experimental data which is larger (or let us say very similar to) than for the surrogate data (Fig. 10), indicating stochasticity. This conclusion is still conflicting with the conclusion we would draw from the critical ratio.

However, for the time series sampled at every fourth point, Figure 11 shows that the forecasting error for the experimental and the surrogate data are similar and that the critical ratio is about two or smaller than two at small forecasting times. This last result, taken together with the preceding results, clearly indicates the stochasticity of the data.

Note that our procedure of removing points in the time series is equivalent to a decrease of the acquisition frequency and somehow in the same spirit than the Theiler procedure [22] of removing neighbours in space which are also neighbours in time. Also, the influence of the sampling time here may be understood as follows: the size of the experimental files is fixed, being limited by the processing time. Therefore, a small sampling time leads us to analyze 

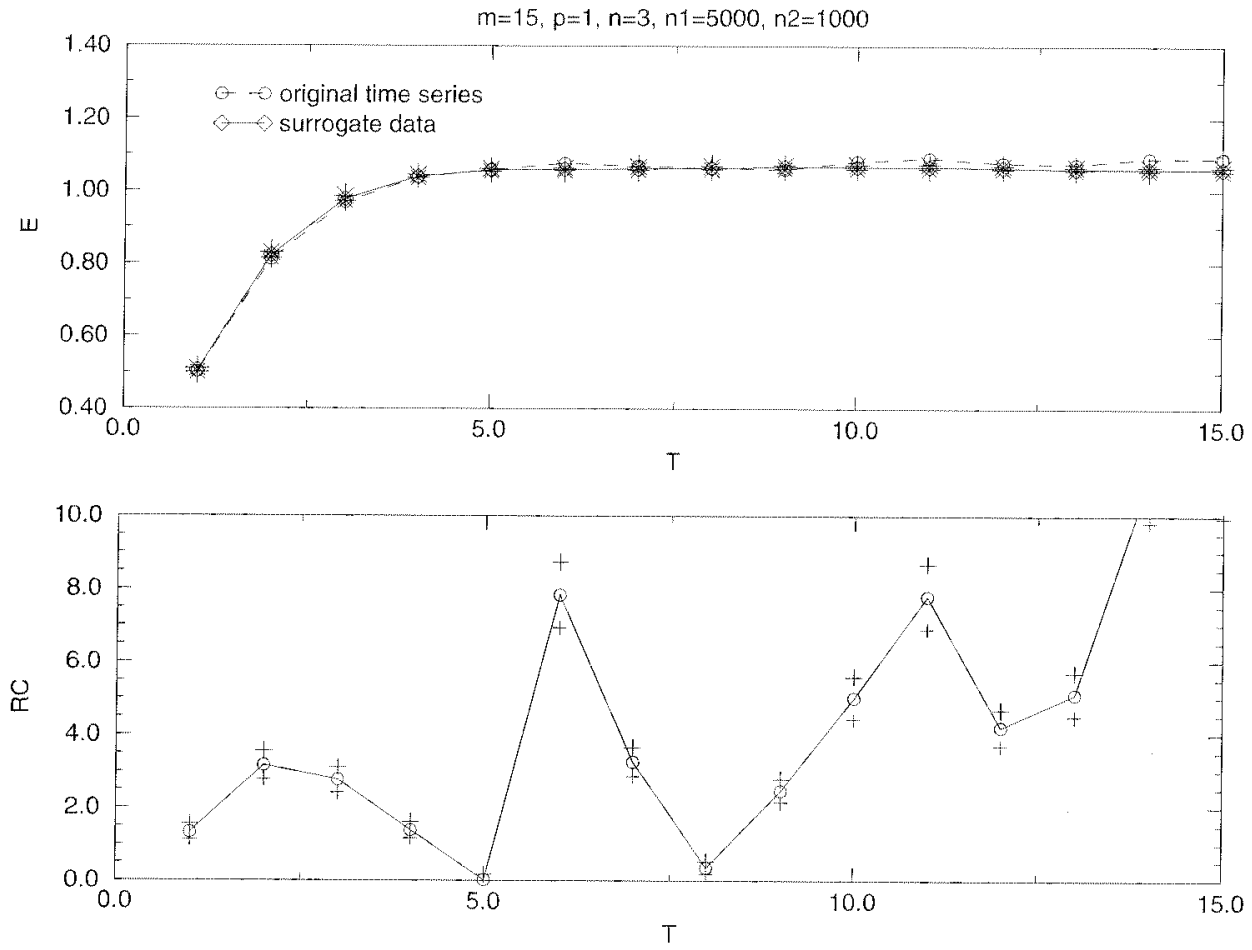

Fig. 11. Forecasting error, and critical ratio, for the same recording, sampled at every fourth point.

a small portion of trajectory. Increasing the sampling time conversely allows us to analyze a larger portion of the trajectory, i.e to obtain more relevant information on the object under study.

4.1. The Effects of Smoothing.--.- Many experimenters use smoothing techniques before processing their data. Let us examine the effects of one of these techniques [27, pp. 495-497], which removes any linear trend, and then uses a Fast Fourier Transform to low-pass the data. The linear trend is reinserted at the end of the process. The amount of smoothing is defined by a constant $K$. Then, the cut-off frequency is given by $f_{\mathrm{c}} \approx n_{\mathrm{q}} / K \times f_{\mathrm{s}}$ where $f_{\mathrm{s}}$ is the sampling rate, and $n_{\mathrm{q}}$ the greater power of two which is smaller than or equal to the size of the data. record.

As shown in Figure 12, the results obtained for a smoothed experimental time series clearly indicate a strong difference for the forecasting error between experimental and surrogate data, and the critical ratio is large. Two conflicting interpretations may then a priori be proposed:

- The signal is deterministic and chaotic. But the non-linear features were masked by the experimental noise.

- The signal is stochastic, but the smoothing procedure introduced low-frequency effects which falsify the results.

We tested these assumptions by applying the same procedure to the analysis of an autoregressive noise. For the untreated process, we obtained a critical ratio smaller than two, and a forecasting error very close to surrogate forecasting error, indicating stochasticity as it should. 

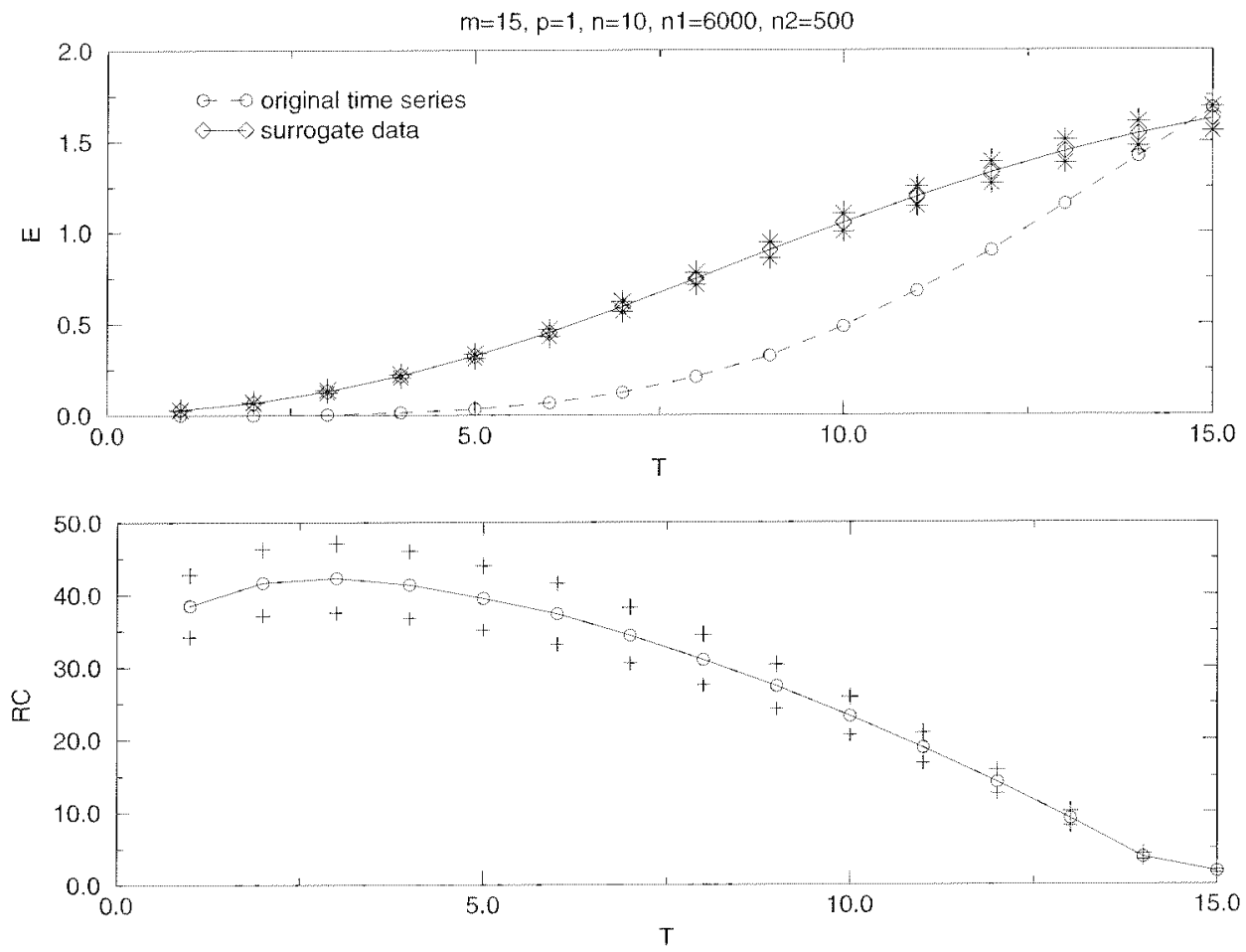

Fig. 12. - Forecasting error, and critical ratio, for a smoothed recording of pressure.

On the contrary, when the time series was smoothed, results displayed in Figure 13 would have lead us to reject the null hypothesis and to conclude to the presence of chaos, which would be a spurious conclusion. Therefore, the use of a noise reduction technique may falsify the results given by the surrogate data method. Our final conclusion on the distinction between noise and chaos for our data is then that the experimental time series measured in the fluidized bed are not generated by a low-dimensional process, but can be the results of a stochastic process. The next step is then to ask whether we can characterize this process.

\section{Hurst Analysis}

Some stochastic processes exhibit long-term statistical dependence, among them the fractal Brownian motions (fBm), which verify [28]:

- the increments $B_{H}\left(t_{2}\right)-B_{H}\left(t_{1}\right)$ and $B_{H}\left(t_{4}\right)-B_{H}\left(t_{3}\right)$ are independent

- an increment $B_{H}\left(t_{2}\right)-B_{H}\left(t_{1}\right)$ follows a Gaussian law, with zero average and a standard deviation proportional to $\left|t_{2}-t_{1}\right|$.

The main property of the $\mathrm{ABm}$ is related to the value of the correlation $C(t)$ between $B_{H}(-t)$ and $B_{H}(t)$, given by:

$$
C(t)=\left(2^{2 H-1}-1\right)
$$

Thus, we can distinguish three cases: 

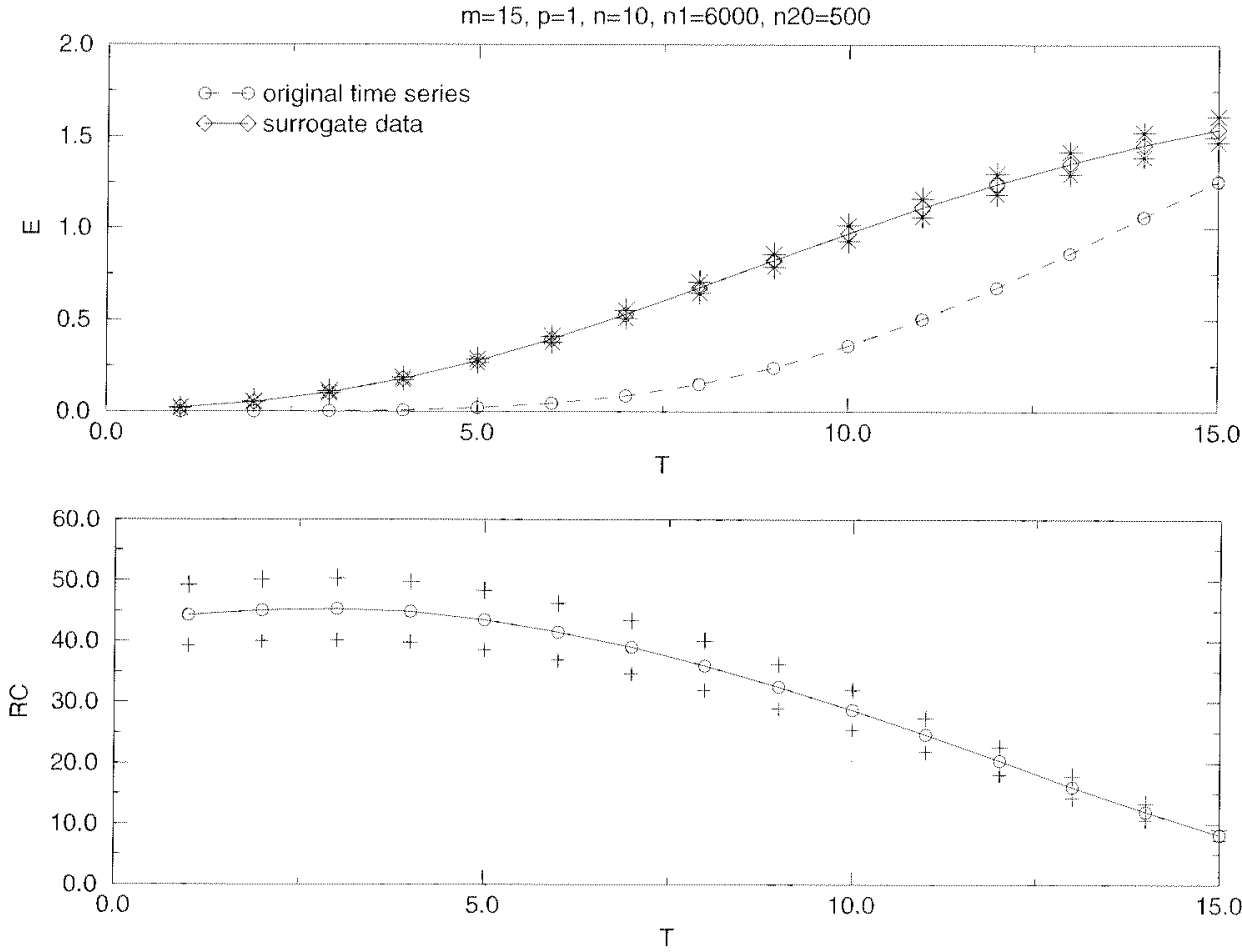

Fig. 13. Forecasting error, and critical ratio, for a smoothed autoregressive noise.

- $H=0.5$. The correlation between $B_{H}(-t)$ and $B_{H}(t)$ is always zero, i.e. the process is a Brownian motion, as defined by Wiener in 1923 [29], with no memory.

- $H>0.5$. The correlation is always positive, i.e, the trends of the graph at the time $-t$ are conserved at the time $t$. Large excursions appear on the curves giving $B_{H}(t)$ versus $t$, and the signal is said to be persistent.

- $H<0.5$. Here exactly the contrary is tme. The signal is said to be antipersistent, and the curves appear to be much more crinkly.

Hurst's analysis $[30,31]$ allows the $H$-value to be deduced from a time series, as follows. First, from the time series $\left(x_{i}\right)$, considered on a total lag time $\tau$, the cumulative departure $X(t, \tau)$ to the average is computed:

$$
X(t, \tau)=\sum_{i=1}^{t} x_{i}-\langle x\rangle_{\tau}
$$

Then we define a range $R$ which is equal to the maximum of the cumulative departure minus its minimum, and depends only on $\tau$ :

$$
R(\tau)=\max _{1 \leq t \leq \tau} X(t)-\min _{1 \leq t \leq \tau} X(t)
$$

Finally, the quantity $R / S$, where $S$ is the mean square deviation of the time series, follows the empirical law:

$$
R / S \propto(\alpha \tau)^{H}
$$




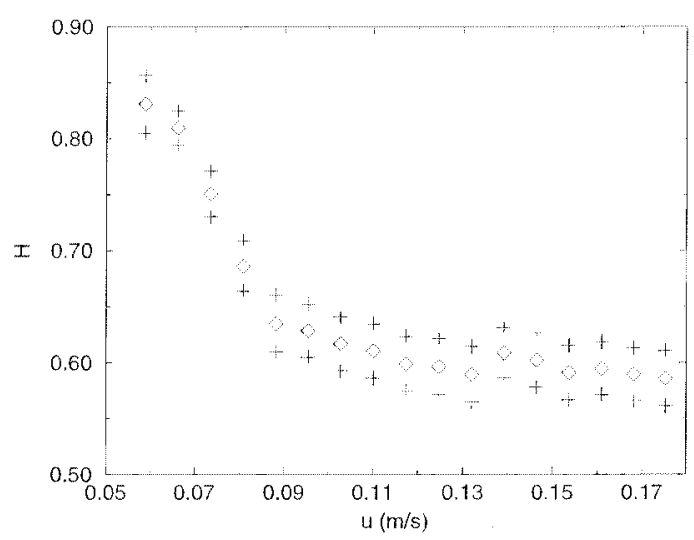

Fig. 14. - Hurst's exponent versus superficial velocity (o), for measurement of right-hand absolute pressure, at point 4 , the symbol $(+)$ indicate error measurement on $H$.

Therefore, by plotting $\log (R / S)$ for different lag times $\tau$, the value of $H$ can be evaluated by linear regression.

5.1. Application to the Experiments. - We calculated the value of $H$, and plotted it versus superficial velocity, as shown in Figure 14. For a small velocity, $H$ is quite large $(\approx 0.8)$, i.e. strong correlations are present in the signal, corresponding to very low frequency effects (the signal is persistent). $H$ decreases with an increase in the superficial velocity, and eventually reaches values around 0.6 , which is not far from the value expected for a Brownian motion. If we consider the fractal dimension associated to $H\left(D_{H}=1 / H\right)$, the latter increases with velocity, representing the fact that the number of bubbles in the bed increases, and that the re-circulation time of the particles decreases.

We noticed that the values found for $H$, for different taps, and for differential pressures as well as for absolute pressures are quite close, so that the difference between them can be due to measurement uncertainties. In this case, it would be possible to associate to a given $H$ a bubbling intensity, and therefore to find a way of controlling the bubbling.

\section{Conclusion}

To analyse pressure measurement in a fluidized bed, we used several techniques to discriminate between noise and chaos. In particular, from results obtained by using a surrogate data method, we concluded that the experimental time series generated by the fluidized bed were probably random, and at least were not low-dimensional chaos. It has been illustrated that much care must be taken to avoid spurious identification of deterministic chaos.

In particular, a naive use of correlation dimension evaluations may be misleading due to the fact that some random processes, characterized by strong correlations, may lead to misleading low-dimension effects. Once our process has been assumed to being random, Hurst's analysis allowed us to suggest that the random process could possibly be, as a first approximation, a fractal Brownian motion characterized by a Hurst exponent $H$. The complicated dynamics exhibited by the fluidization process may then be characterized by a single number. The use of such a synthetic number could allow the identification of fluidization regimes.

It should however be noted that our study has been carried out in terms of a noise/chaos 
distinction. In many cases, both aspects are mixed. Therefore, if there exists a deterministic component in our signals, a more refined conclusion would be that this component conld not be detected by our analysis, i.e. the dynamics of the fluidized bed is dominated by randomness.

\section{References}

[1] Van Den Bleek C.M. and Schouten J.C., Chem. Eng. J. 53 (1993).

[2] Tam S.W. and Devine M.K., Is there a strange attractor in a fluidized bed ?, in Measures of Complexity and Chaos, N.B. Abraham et al. Eds., (Plenum Press, New York).

[3] Provenzale A., Smith L.A., Vio R. and Murante G., Physica D 58 (1992) 31-49.

[4] Meunier-Guttin-Cluzel S., Maheu B., Gouesbet G., Physica D 58 (1992).

[5] Rapp P.E., Albano A.M., Schmah T.I., Farwell L.A., Phys. Rev. E 47 (April 1993).

[6] Theiler J., Eubank S., Longtin A., Galdrikian B. and Farmer J.D., Using surrogate data to detect nonlinearity in time series, in Nonlinear prediction and modeling, M. Casdagli and S. Eubanks Eds., (Addison-Wesley, 1992).

[7] Theiler J., Phys. Rev. A 34 (1986) 2427-2432.

[8] Thioye M., Darrigo R. and Gonesbet G., Monte-Carlo simulation of bubbling fluidized beds, in Instabilities in Multiphase Flows, G. Gouesbet and A. Berlemont Eds., (Plenum Press, 1993).

[9] Letaief N., Distinction bruit/chaos appliquée à l'étude d'un lit fluidisé bouillonnant, Thèse de Doctorat de l'Université de Rouen, France (Janvier 1990).

[10] Fan Z., Chen G.T., Chen B.C. and Yuan H., Powder Tech. 62 (1990) 139-145.

[11] Neogi D., Fan L.T., Yutani N., Nassar R. and Walawender W.P., Apl. Stoch. Models Data Analysis 4 (1988) 13-34.

[12] Kage H., Iwasaki N., Yamaguchi H. and Matsuno Y., J. Chem. Eng. Jpn 24 (1) (1991).

[13] Saxena S.C. and Rao N.S., Energy 15 (1990) 489-497.

[14] Huang Y.W., Fan L.T., Song J.C. and Yutani N., Pressure fluctuation in a gas-solid fluidized bed in a screen.

[15] Drahos J., Bradka F. and Puncochar M., Chem. Eng. Sci. 47 (1992) 4069-4075

[16] Grassberger P. and Procaccia I., Physica D 9 (1983) 189.

[17] Gouesbet G., Phys. Rev. A 42 (1990) 5928-5945.

[18] Farmer J.D and Sidorowich J.J, Phys. Rev. Lett. 59 (August 1987).

[19] Sugihara G. and May R.M., Nature 334 (Avril 1990).

[20] Wales D.J., Nature 350 (April 1991).

[21] Tsonis A.A. and Elsner J.B., Nature 358 (July 1992).

[22] Theiler J., Eubank S., Longtin A., Galdrikian B. and Farmer J.D., Physica D 58 (1992).

[23] Buzug Th. and Pfister G., Phys. Rev. A 45 (Mai 1992).

[24] Aleksie Z., Physica D 52 (1991).

[25] Liebert W. and Schuster H.G., Phys. Lett. A 142 (December 1989).

[26] Buzug Th. and Pfister G., Europhys. Lett. 13 (December 1990).

[27] Press W.H, Flannery B.P., Teukolsky S.A. and Vetterling W.T., Numerical Recipes (Cambridge University Press, 1986).

[28] Mandelbrot B.B. and van Ness J.W., SIAM Rev. 10 (October 1968).

[29] Wiener N., J. Math. Phys. Mass. Inst. Technol. 2 (1923).

[30] Hurst H.E., Trans. Am. Soc. Civ. Eng. 116 (1951).

[31] Feder J., Fractals (Plenum Pub. Corp., New York, 1988). 
